\title{
The Best among Six Strategies for Selecting a Minute Target and the Determination of the Minute Maximum Size of the Targets on a Pen-Based Computer
}

\author{
Xiangshi Ren and Shinji Moriya \\ Department of Information and Communication Engineering \\ Tokyo Denki University \\ 2-2 Kanda-Nishikicho, Chiyoda-ku, Tokyo 101, JAPAN \\ $+81-3-5280-3335$ \\ \{ren,moriya\}@c.dendai.ac.jp
}

\begin{abstract}
This study concentrates on finding the relationship between target-pointing strategies and target sizes. Concretely, an evaluation experiment was performed in which the experimental system used five kinds of targets $(1,3,5$, 7 and 9 dot diameter circles in respectively, $0.36 \mathrm{~mm}$ per dot), eight pen-movement-directions and six strategies of systematic election on a pen-based computer. The results showed that the "Land-on2" strategy was the best strategy for selecting a minute target, in terms of error rates, selection time and subjective preferences. "The minute maximum size" of 5 dots was also determined to be the largest size among the targets which had a significant main effect on the six strategies in terms of error rates.
\end{abstract}

KEYWORDS input devices, pen-based input, target-pointing strategies, minute targets, the minute maximum size.

\section{INTRODUCTION}

Pen-based computers operated only with pens are attracting attention in many fields. Yet, many problems remain. One of the main goals is to achieve an efficient pen input user interface. The pen-based user interface is very different from other user interfaces. In portable systems, it is much easier to do all the operations with a pen than with a keyboard or a mouse. In portable penbased systems selection is used more often than data input. "Selection" means using a pen to select menus. data (one character of the text or graphic segment, etc.), and ranges, etc. In this paper, these are called "targets".

It is predicted that the target size on pen-based systems will decrease while the amount of information on the screen will increase. Up to now, there have been only a few leading studies on the selection of a small target (Sears and Shneiderman, 1991; Potter, Weldon and Shneiderman, 1988; McClintock and Hoiem, 1993). Sears and Shneiderman (1991) tested three selection devices; touch screen, touchscreen with stabilization and mouse. The task was the selection of rectangular targets of $1,4,16$ and 32 pixels per side. Their results showed that a stabilized touchscreen was effective for reducing the error rates when selecting small targets. Potter, Weldon and Shneiderman (1988) compared three touchscreen strategies, land-on, first-contact and takeoff. The results showed that the take-off strategy had the lowest error rate and highest satisfaction for users. However, experimental targets were limited to only one size (6.4 $\mathrm{mm} \times 6.4 \mathrm{~mm}$ square). McClintock and Hoiem

Human-Computer Interaction: INTERACT'97 S. Howard, J. Hammond \& G. Lindgaard (editors)

Published by Chapman \& Hall CIFIP 1997 


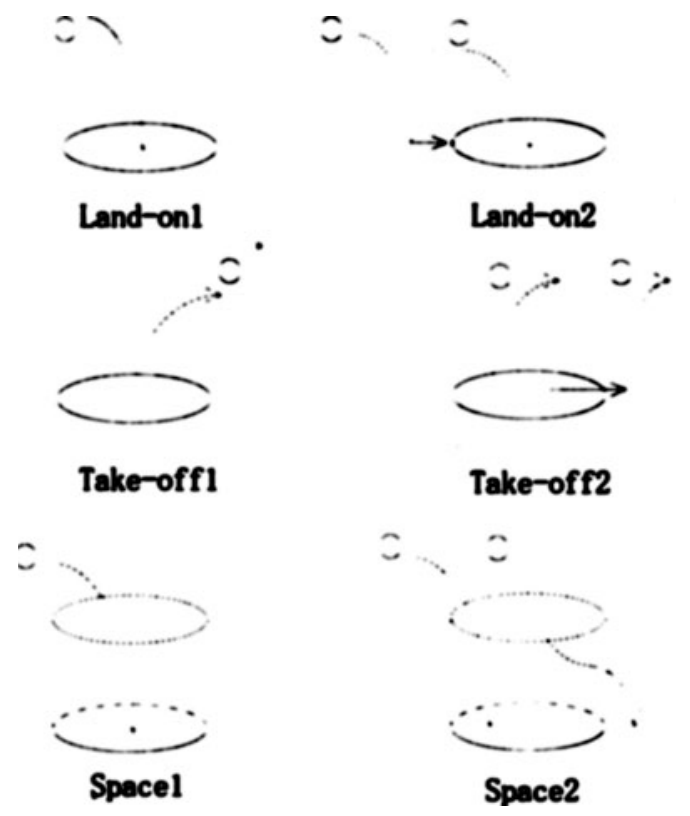

Figure 1. Six strategies used by the experiment.

(1993) conducted a study to determine the smallest optimal size for targets in a pen-based system. Eight kinds of targets were used but the study did not report on any target-pointing strategies. Some findings concerning the pointing strategies have been described elsewhere (see, for example, Sears, Plaisant and Shneiderman, 1992; Buxton, 1990). However, current target-pointing strategies on pen-based computers are mostly only imitations of selection techniques for mouse and touchscreen devices. Investigations have not been conducted which focus on strategies suitable for selecting minute targets in pen-input systems. Based upon our previous experiment (Ren and Moriya, 1995), this study focuses on the relationships between target-pointing strategies and target sizes.

\section{THE SIX STRATEGIES AND THE REASON FOR USING THEM}

\subsection{The Six Strategies}

The six strategies are illustrated in Figure 1. We used an electromagnetic tablet. When the pen-tip is within a given height above the tablet surface $(1 \mathrm{~cm})$, the coordinates $(x, y)$ of the pen-tip are able to input data. Thus, even though the menu on the screen is 2 dimensional (2D), it can be highlighted or selected when the pen is above the tablet surface (within $1 \mathrm{~cm}$ ). This means that the menu can be expressed as a 3 dimensional (3D) target.

The oval and the cylinder shown in Figure 1 illustrate targets on the pen computer screen. The oval shows that the target is a 2D target. The cylinder shows that the target is a 3D target. That is, the circle with a solid line is at the bottom of the 3D target. Some responses will take place when the pen is in the cylinder. It is important to note that although the illustration (Figure 1) shows circular targets, the shape of the target has no definitive bearing on this discussion.

The arrow in Figure 1 shows the movement of the pentip. A dashed line arrow means the pen-tip is above the screen and a solid line arrow shows that the pen-tip is on the screen. $\bigcirc$ shows that the target is selected by the movement of the pen.

- Land-on1: the pen approaches from above. The target is selected only momentarily at the time the pen makes contact with the screen in the target area.

- Land-on2 is an extension of the Land-on1 strategy. Here also the target is selected when the pen touches it for the first time, but in this case the pen lands outside the target area before moving into it.

- Take-off1: the target is highlighted only while the pen is touching it. The selection is made at the moment the pen is taken off the target.

- Take-off2 is an extension of the Take-offl strategy. The target is highlighted only while the pen is in contact with it, however the selection is made when the pen is removed from any point on the screen either inside or outside the target area.

- Spacel: the pen approaches from above. The target is highlighted while the pen is within the $1 \mathrm{~cm}$ high cylinder above the target. Selection is made at the 
moment the pen makes initial contact with the target area (i.e. inside the bottom circle).

- Space2 is an extension of the Spacel strategy. The target is highlighted while the pen is within the $1 \mathrm{~cm}$ high cylinder above the target. After highlighting, the selection is made when the pen makes contact with any point on the screen either inside or outside the target area.

Land-on 1 and Take-offl strategies are already in common use. The Land-on2 strategy corresponds to the first-contact strategy (Potter, Weldon and Shneiderman, 1988). However, the Take-off2, Spacel and Space2 strategies were new strategies designed for this experiment.

\subsection{Characteristics of the Six Strategies and their Classification, and Reasons for the Determination of those Six Strategies}

What characteristics do these various strategies have? What criteria were used to select the six strategies used here? Regarding the above questions, we concentrated on the six conditions created by the pen parameters. They are: contact with the screen, remove from the screen, contact inside the target, contact outside the target, target highlighted and target not highlighted.

Targets exist both as planes (2D) and as solid bodies (3D). Here, the 2D strategies are the Land-on1, Landon2, Take-off1 and Take-off2 strategies. The 3D strategies are the Spacel and Space2 strategies.

Contact and removal of the pen were considered as movements between the 2D plane and 3D space. Pen contact involves a movement from $3 \mathrm{D}$ to $2 \mathrm{D}$, while removal involves a movement from $2 \mathrm{D}$ to $3 \mathrm{D}$. These changes were considered to be suitable conditions for the subject to recognize and confirm the moment of target selection. The strategies in which selection was made by contact with the screen (Land-on1, Land-on2, Space1 and Space 2 strategies) were named On strategies. The strategies in which selection was made by removal from the screen (Take-off1 and Take-off 2 strategies) were named Off strategies. Where the target existed on the 2D plane, both the On and Off strategies were deployed. Where the target existed in 3D space, only the On strategies were used, assuming that the pen was approaching the target from above.
We considered the movement of the pen into and out of the target from the perspective of the user's eyes and ears. When the pen moved into or out of the target, users could confirm whether or not the target was highlighted. Those strategies in which selection was made by contact within the target area were named In strategies (the Land-on1, Take-off1 and Spacel strategies). On the other hand, those strategies in which selection was made by contact either inside or outside the target were named In-Out strategies (Land-on2, Take-off2 and Space2 strategies).

Those strategies in which selection was made when the pen was removed from the surface of the target or from above the target after visual confirmation, were named 2Stage strategies (the Take-off1, Take-off2, Spacel and Space2 strategy). Those strategies in which visual confirmation was not possible were named 1Stage strategies (Land-on1 and Land-on2 strategies).

\section{METHOD}

\subsection{Subjects}

Nineteen subjects ( 14 male, 5 female; 18 right-handed, 1 left-handed), all university students, were tested for the experiment. Their ages ranged from twenty-one to twenty-three years old. Seven people had previous experience with pen-input systems, while the others had no experience.

\subsection{Equipment}

The hardware used in this experiment was: a tabletcum-display (HD-640A, WACOM Co.), a stylus pen (SP-200A, WACOM Co.), and a personal computer (PC9801-DA, NEC Co.). The space resolution of the tablet input was $0.05 \mathrm{~mm} /$ point. The height of the liquid crystal screen was $144.0 \mathrm{~mm}$ and the width was 230.4 $\mathrm{mm}$. The liquid crystal display resolution was 400 dots high and 640 dots wide. 1 dot was about $0.36 \mathrm{~mm}$.

\subsection{Procedure}

First we explained the experiment to each subject and they each had 20 practice trials. A message "Select a target as quickly and accurately as possible using the strategy" was displayed on the screen of the experimental tool when the experiment started. 


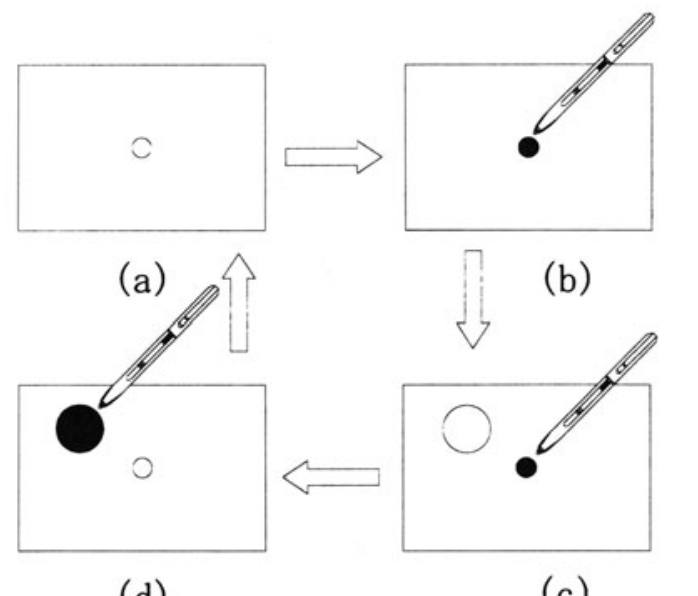

(d)

(c)

Figure 2. Steps in selecting a target. (a) Initial position, (b) Pointing at the initial position, (c) Display of a target, (d) Target selection.

Figure 2 shows a typical display which would appear when a target was being selected using any one of the strategies. (a) initial position: a circular initial position was displayed at the center of the screen. The initial position was the place where the pen would be pointed immediately before beginning the selection procedure. The subject had been told which strategy he/she was to use and how many trails he/she had to do. (b) Pointing at the initial position: the subject pointed to the initial position with the pen. (c) Display of a target: the target was displayed with the size and direction changed at random (See 3.4.). The parameters of the target (position and size) were randomly selected by the computer. Targets of a particular size were never displayed in the same position twice. The distance between the initial position to the target was constant at 131 dots. (d) Target selection: the subject would receive a message on the screen to indicate whether he/she had made a successful selection or not. (e) The subject then repeated (a) and (d) above. (f) End of selection: a message indicating the end of the experiment was displayed when the subject had completed the task.

The strategies were not mixed. In a given trial each subject used only one strategy.

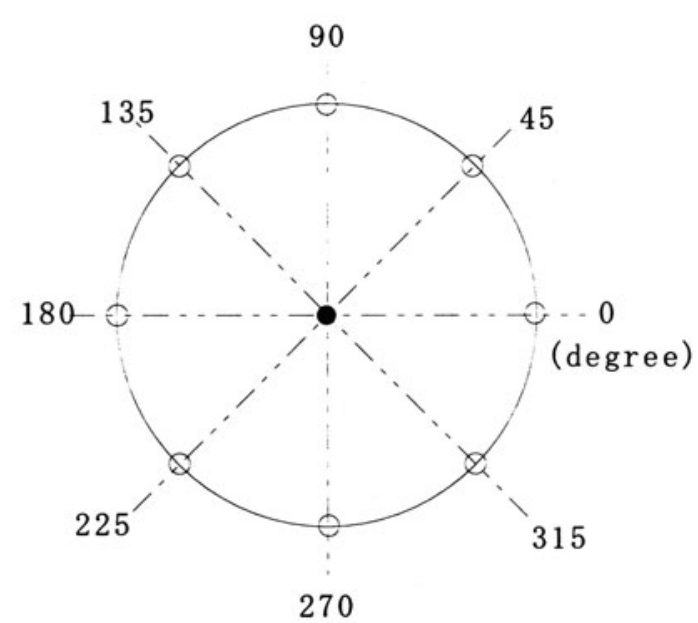

Figure 3. An example of arranging a target. - : Initial position, $\bigcirc$ : Position of a target display.

\subsection{Design and Data Processing}

Figure 3 shows the eight possible target positions.

Size of target: all the targets were circular. To examine the relationship between target size and the six strategies we used five target sizes in all trials $(1,3,5,7$ and 9 dot diameter circles).

Distance to target: the distance to the target was the radius of a circle in which the center point was the initial position. The distance of 131 dots $(4.70 \mathrm{~cm})$ was determined by a preliminary experiment. This was the average distance that ten subjects could extend the pen to the target.

Pen-movement directions : eight directions were used. They were at $0,45,90,135,180,225,270$ and 315 degrees from the initial position.

The subject had a total of 60 trials for each strategy. These consisted of 20 practice trials and 40 test trials $(=5$ target sizes $\times 8$ directions).

A break was taken at the end of each strategy trial. Whenever the subject felt tired he/she was allowed to take a rest. Each subject completed 240 test trials $(=6$ strategies $\times 40)$. In each strategy 760 test trials (= 19 subjects $\times 40$ ) were completed. The order for the six strategies was different for each of the nineteen subjects. 
The data for each strategy were recorded automatically as follows:

(i) Presence or absence of error when a target was selected: one selection was a continuous operation from the approach of the pen to the target until the removal of the pen from the tablet surface. Feedback to the subject indicated whether the selection was successful or not. In either case, the subject could not cancel the selection.

(ii) Position and size of the target displayed.

(iii) The time lapsed between display of the target and the moment when the pen contacts the screen.

(iv) The time lapsed between contact with the target and removal from the screen.

(v) The time lapsed between contact with the screen and contact with the target.

These times were measured to an accuracy of $10 \mathrm{~ms}$ using a special program.

Data as defined in item (iii) was recorded for the Land-on1, Spacel and Space2 strategies. Data as defined in item (v) above was recorded for the Land-on2 strategy. Data as defined in item (iv) above was recorded for the Take-off1 and Take-off2 strategies.

\subsection{Subjective Evaluation}

The subjects were questioned about their preferences after they ended each trial. The first question was: "For the strategy tested just now, how do you rate P? Please answer on a 1-to-9 scale (123456789).". P consisted of six subquestions regarding selection accuracy, selection speed, selection ease, learning ease, satisfaction and desire to use. The second question was: "Which direction was most comfortable for selecting the target in the above strategies?". The subject marked his/her preferences on Figure 3.

\section{RESULTS}

To determine the effect, in all six strategies, of target size and pen-movement-direction on error rates, selection times and subject preferences, an ANOVA with repeated measures was performed.

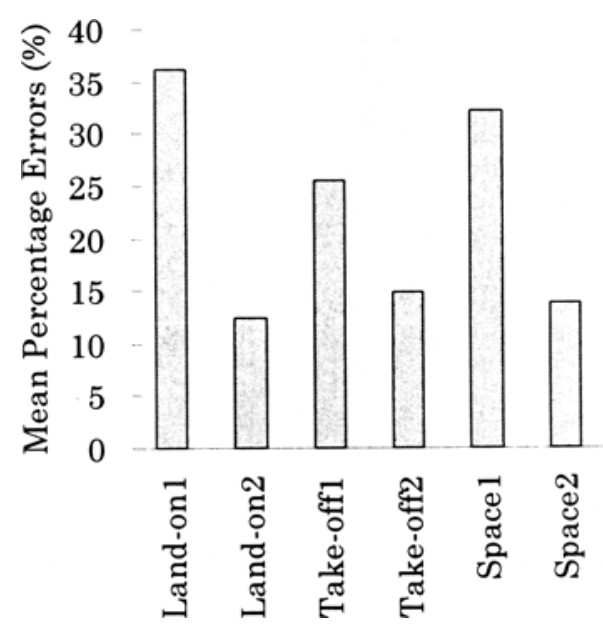

Figure 4. The error rates for each strategy.

Error rates were determined by dividing the number of errors by the total number of selection attempts. Selection time was the time required to select the target correctly.

\subsection{Error Rates}

The error rates for each of the six strategies is shown in Figure 4. There was a significant difference for the six strategies, $F(5,108)=9.76, p<.01$. This means that the difference in error rates was due to the difference in the various strategy operations.

To investigate why there was a significant difference for the six strategies, analyses were conducted between each of four strategy groups. The results showed that there were no significant differences $(p<.05)$ between the On and Off strategies, or between 2D and 3D strategies, or between 1Stage and 2Stage strategies.

However, there was a significant difference between the In and In-Out strategies, $F(1,36)=18.1, p<.001$. When the average values of In and In-Out strategies were compared, the In strategies produced a $31 \%$ error rate and the In-out strategies produced a $14 \%$ error rate. Figure 5 shows that the In-Out strategies had lower error rates than the In strategies in every target size (1, 3, 5, 7 and 9 dots). The error rates for the Land-on2 strategy 


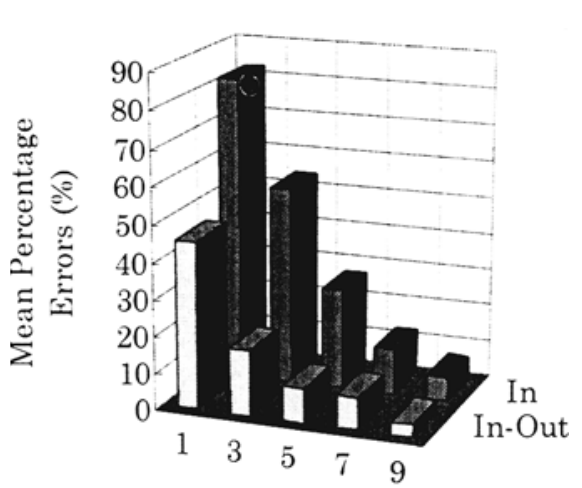

Target size

(dots in diameter, 0.36 mm per dot)

Figure 5. Error rates per target size for the In strategies and the In-Out strategies.

were the lowest among the six strategies (see Figure 4). But there was no significant difference in the total numbers of errors for the five target sizes $(1,3,5,7$ and 9 dots) for the Land-on2, Take-off 2 and Space2 strategies in the In-Out strategy group, $F(2,54)=0.19$. The error rates for these three strategies did not differ for target sizes of 1,3 and 5 dots, $F(2,54)=0.07$.

However, when all six strategies were compared, there were significant differences in error rate, in terms of the target sizes of 1, 3 and 5 dots. On the other hand, there were no significant differences in 7 or 9 dots between the six strategies $(p<.05)$.

Regarding the pen-movement directions, significant differences were seen in the six strategies for each direction $(p<.01)$. This means even when pen-movement directions were different, there were no changes in error rates for the six strategies.

\subsection{Selection Time}

The selection time for each of the six strategies is shown in Figure 6. There was a significant difference between the six strategies, $F(5,108)=3.17, p<.05$. This means selection times varied according to the strategy
180

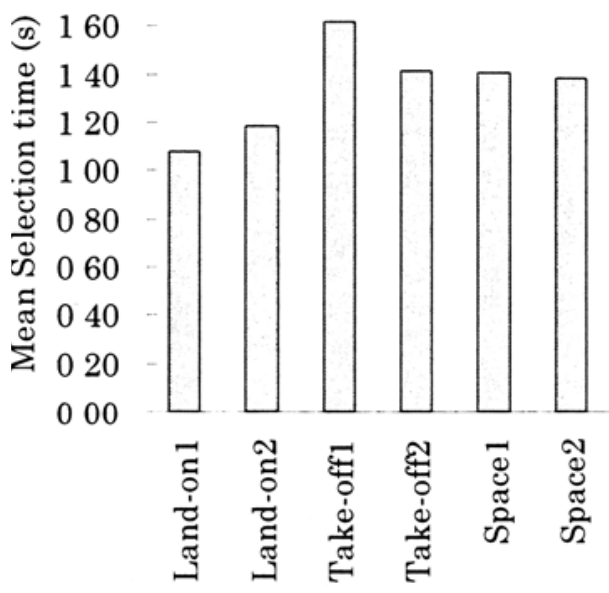

Figure 6. The selection time for each strategy.

used. This indicates that the strategies influenced the selection times.

We investigated the cause of the significant differences in the six strategies. Comparisons between each of the four strategy groups showed there were significant differences between the On and Off strategies, $F(1,36)=$ $242.9, \mathrm{p}<.0001$, and between the 1Stage and 2Stage strategies, $F(1,36)=8.37, p<.01$. There were no significant differences between the $2 \mathrm{D}$ and $3 \mathrm{D}$ strategies, or between the In and In-Out strategies $(p<.05)$. The average times of the On and Off strategies and the 1Stage and 2Stage strategies, showed that the On strategies (1.26 s) were faster than the Off strategies (2.41 s) and 1Stage strategies $(1.13 \mathrm{~s})$ were faster than 2Stage strategies (1.46 s).

There was a significant difference in selection time between the six strategies. There were significant differences for every target size ( 1 dot, $p<.05 ; 3,5,7$ and 9 dots, $\mathrm{p}<.01)$. The differences in selection times caused by variations in the target size were constant.

Regarding the pen-movement directions; there were significant differences for the six strategies in the penmovement-directions $0,45,90,135,225$ and 270 degrees $(p<.05)$. On the other hand, there were no significant differences for the six strategies in the directions 180 or 315 degrees $(p<.05)$. 


\subsection{Subject Preferences}

The analysis of the questionnaire showed a significant main effect on subject preferences for each of the six strategies, $F(5,30)=258.5, p<.0001$. In addition there was a significant difference in subject preferences between the In and In-Out groups of strategies. $\mathrm{F}(1,10)=$ $803.1, \mathrm{p}<.0001$. This agreed with the test results. Moreover, among three of the strategies (Land-on2, Take-off2 and Space2 strategies) there was also a significant difference in preferences, $F(2.15)=7.90, p$ $<.01$. Rated on a scale of 1-to-9 the Land-on2 strategy was the most preferred $($ mean $=8.0)$.

There was a significant difference in subject preferences regarding pen-movement directions, $F(7,40)$ $=17.5, \mathrm{p}<.0001$, however, the preferences marked by the subjects in Figure 3 revealed that 225 degrees was the most preferred direction $($ mean $=10.5)$.

\section{DISCUSSION}

Of the six strategies, the Land-onl and Take-off 1 strategies are used most often. However. according to our results, we found the Land-on2 strategy was the most effective for selection of a minute target.

As for selection time, a significant difference appeared between 1Stage and 2Stage strategies, and between On and Off strategies. This difference did not depend on target size or pen-movement-direction (180 and 315 degrees were excluded). On strategies were selected faster than Off strategies. 1Stage strategies were selected faster than 2Stage strategies. It is believed that of the six strategies tested, Land-on 1 and Land-on2 strategies were the fastest in selection times. This is because they are both On strategies and 1Stage strategies. In addition, the Land-on2 strategy had lower error rates. Thus we have determined that the Land-on2 strategy was the best strategy for selection of a minute target in terms of selection time and error rates. In addition, there was no significant difference in selection times between the Land-on 1 and Land-on2 strategies. $F(1,36)=0.53$. Furthermore the subjects also rated the Land-on2 strategy most highly.

Comparisons of each classified group revealed a significant difference in error rates between the In and the In-Out strategies. This implies that differences in error rates were independent of target size (1, 3 and 5 dots) and pen-movement-directions for these strategy groups. We concluded that the In-Out strategy group was the most effective for selecting a minute target.

Regarding error rates, there was no significant difference in the results when the targets were large ( 7 or 9 dot). On the other hand, there were significant differences for the six strategies when the target sizes were minute ( 1,3 and 5 dots). The reason for this lies in the difference between the In and In-Out strategies. The In strategies were adequate when choosing large targets because there was no strain on the subject. When choosing a minute target, the selection was not accurate using the In strategies only, thus the In-Out strategies were more effective. for example, where targets could be selected by directly pressing the target with the pen. In the case of a minute target, it was necessary for the subjects to confirm visually whether or not the pen was inside the target area while either making contact or taking off. Using the In strategies. selection is completed at the moment of contact or take off. On the other hand, the InOut strategies do not require visual confirmation of the pen position vis-a-vis the target. When selecting a minute target with the In-Out strategies, one can direct the pen from a point on the surface of the target after landing on the screen, even if it is impossible to place the pen directly inside the target (as in the Land-on2 strategy). In the case of the Take-off2 strategy, selection was achieved even if a parallax on hand-movement occurred. In the questionnaire subjects overwhelmingly preferred the InOut strategies. From this it is clear that the In-Out group of strategies is superior.

We have observed the following concerning the strategies of selecting a minute target. When a minute target on the 2D tablet surface is used (e.g. a pressure perception type tablet like ZAURUS, SHARP Co.). it is possible to condude: if there are no other targets near a minute target (e.g. a point or width of a line with 1 to 5 dots on a graphic screen or a minute icon on a small pen-input system), the Land-on2 and Take-off2 strategies in the In-Out group are most effective for selecting the target. The Land-on2 strategy is especially effective when selection speed is considered.

When selecting target sizes of more than 5 dots. the selection performance became identical even when using different strategies. We concluded that 5 dots $(1.80 \mathrm{~mm}$ circle in diameter) was the largest size among the targets. 
in which there was a significant main effect on error rates for the six strategies. We called this "the minute maximum size". Therefore, 5 dots was the minute maximum value from the experimental results.

In Fitts' law (Fitts, 1954), ID $=\log _{2}(2 D / W)$, ID is an abbreviation of Index of Difficulty of pointing, D is the distance, and $\mathrm{W}$ is the width of the target. If $\mathrm{D}$ is a constant, $\mathrm{ID}_{1}=\log _{2}\left(2 \mathrm{D} / \mathrm{W}_{1}\right), \quad \mathrm{ID}_{2}=\log _{2}\left(2 \mathrm{D} / \mathrm{W}_{2}\right), \ldots, \mathrm{W}_{1}$, $\mathrm{W}_{2}, \ldots>5$ dots, then, as far as our results are concerned, there are no significant differences among $\mathrm{ID}_{1}, \mathrm{ID}_{2}, \ldots$, $\mathrm{ID}_{\mathrm{n}}$. In other words, when selecting target sizes of more than 5 dots, differences in the ID (error rates) due to target size will disappear. Thus, we clarified the boundary value of the target size which controlled the difficulty of strategies. When a target is less than 5 dots, the strategy should be designed in line with our findings. This work defines values by which not only pen-based devices but also other devices may be researched.

In this experiment, circular targets were used so that the distance which the pen reaches to the edge of a target remained constant in all directions. In another experiment (Ren and Moriya, 1995), it was found that there were significant differences in terms of error rates and selection times when various distances were used; 39 , 131 and 160 dots. By using only one of the three distances we were able to decrease the number of trials in this experiment. 131 dots was chosen as the distance because we had determined that it was the maximum fingermovement-distance when the wrist was in a fixed condition using a portable pen-input systems.

Regarding pen-movement-directions, a vector of 225 degrees was preferred by most of the subjects. We assumed that the reason was that this direction was a position from which right-handed subjects could see most. Moreover, a significant difference was not seen for the six strategies using directions 180 and 315 degrees. Wrist angle and sight angle are factors to be considered. In future research we will consider the relationships between pen-movement-directions and target-pointing strategies.

In this experiment, we focused only on the selection of a single target. We will attempt to compare the six strategies in multi-targets environments in future studies. It has been reported elsewhere that differences in the target shapes influence the selection time (Sheikh and Hoffmann, 1994). It is necessary to examine other target shapes in order to compare them with the results pertaining to circular targets used in this experiment. It is also necessary to investigate the relationships between strategies and target shapes and to find strategies which are suitable for specific shapes. Attention should be given to whether or not those shapes change the minute maximum size of a target area.

\section{REFERENCES}

Ren X. and Moriya S. (1995). The concept of various pointing strategies on pen-based computers and their experimental evaluation, Proceedings of the Eleventh Symposium on Human Interface (Kyoto, Japan), pp.565-574.

Sears A. and Shneiderman B. (1991). High precision touchscreens: Design strategies and comparisons with a mouse, International Journal of Man-Machine Studies, Vol.34, No.4, pp.593-613.

Potter R., Weldon L. and Shneiderman B. (1988). Improving the accuracy of touch screens: An experimental evaluation of three strategies, Proceedings of ACM the CHI'88 Conference on Human Factors in Computing Systems, pp.27-32.

Buxton W.(1990). A three state model of graphical input. In D. Diaper et al. (Eds.), Human-Computer Interaction -INTERACT '90, pp.449-456.

Sheikh I. and Hoffmann E. (1994). Effect of target shape on movement time in a Fitts task. Ergonomics, Vol.37, No.9, pp.1533-1547.

Fitts P.M. (1954). The information capacity of the human motor system in controlling amplitude of movement, Journal of Experimental Psychology, Vol.47, No.6, pp.381-391.

McClintock M. and Hoiem D. (1993). Minimal target size in a pen-based system, In Abridged Proceedings of 5th International Conference on Human-Computer Interaction, Elsevier Science Publishers B.V., p.243.

Sears A., Plaisant C. and Shneiderman B. (1992). A new era for high precision touchscreens, Advances in Human-Computer Interaction, Vol.30, Ablex, Norwood, NJ, pp.1-33. 